\title{
High-resolution mass spectrometry
}

\author{
Hans H. Maurer • David C. Muddiman
}

Published online: 10 April 2012

(C) Springer-Verlag 2012

High-resolution mass spectrometry (HRMS) using doublefocusing mass analyzers was established in chemistry laboratories in the 1960s. Coupled to a direct insertion probe or to gas chromatography, it revolutionized the elemental analysis of organic compounds. It also became a powerful tool in bioanalytical research providing high selectivity and specificity. However, modern benchtop GC-MS instruments cost significantly less and have been coupled to the faster scanning quadrupole or ion-trap mass analyzers, largely displacing GC-HRMS in most fields.

Modern LC-MS instruments using electrospray ionization have many applications in bioanalytical laboratories and in "omics" research. Manufacturers, for arguably the first time, recognized a sizeable market for HRMS. In the past few years, robust GC-MS and, particularly, LC-MS equipment, mainly using time-of-flight or Fourier-transform mass-analyzer technology, are becoming increasingly affordable by many laboratories. This is also apparent from the remarkable increase in the number of publications.

Published in the special issue High-Resolution Mass Spectrometry with guest editors Hans H. Maurer and David C. Muddiman.

H. H. Maurer $(\bowtie)$

Department of Experimental and Clinical Toxicology, Institute of Experimental and Clinical Pharmacology and

Toxicology, Saarland University,

66421 Homburg, Saar, Germany

e-mail: hans.maurer@uks.eu

D. C. Muddiman

Dabney Hall, North Carolina State University,

2620 Yarbrough Drive,

Raleigh, NC 27695-8204, USA

D. C. Muddiman

Department of Chemistry,

W.M. Keck FT-ICR Mass Spectrometry Laboratory,

North Carolina State University,

Raleigh, NC 27695, USA
Therefore, the objective of this special volume is to give an overview of the current use of HRMS in the life sciences. Four review articles critically assess the use of HRMS in clinical and forensic toxicology, doping control, drug metabolism studies, food analysis, and the environmental sciences. This overview is followed by four original papers with new results from application of HRMS to drug screening, doping control, glycomics, and proteomics.

We hope you find this special issue an invaluable source of information; it is intended for all readers with an interest in application of HRMS in clinical and forensic toxicology, doping control, drug metabolism, food analysis, environmental sciences, and proteomics and related fields. We thank all authors for submitting their interesting contributions for this special issue, the referees for their critical but constructive comments, and the editorial office and the editors for their friendly cooperation. 


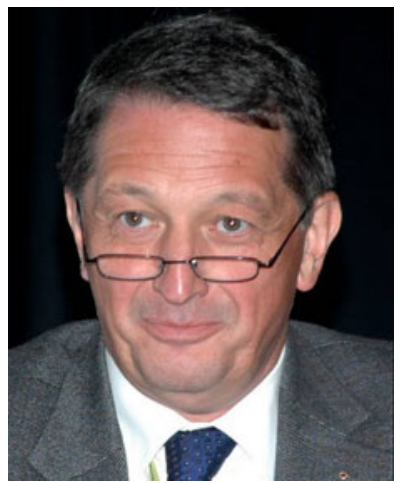

Hans H. Maurer is the Head of the Department of Experimental and Clinical Toxicology of Saarland University in Homburg. He has published over 200 original papers and over 20 invited reviews on his main two areas of research, analytical toxicology (GC-MS, LC-MS) and metabolism of xenobiotics. He received several international scientific awards for his outstanding scientific work, and in 2007 he was awarded the title of Doctor Honoris Causa (honorary doctorate) by the University of Ghent, Belgium.

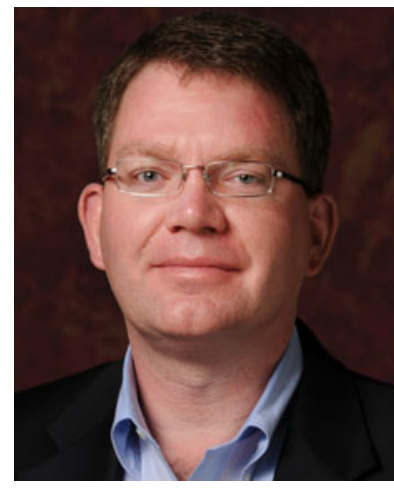

David C. Muddiman is currently a Professor of Chemistry and Founder and Director of the W.M. Keck FT-ICR Mass Spectrometry Laboratory at North Carolina State University in Raleigh, NC, USA. His group has presented over 350 invited lectures and presentations at national and international meetings, has published over 195 peerreviewed papers, and has been awarded three US patents. He is the recipient of the 2010 Biemann

Medal of the American Society for Mass Spectrometry, the 2009 NCSU Alumni Outstanding Research Award, the 2004 ACS Arthur F. Findeis Award, the 1999 American Society for Mass Spectrometry Research Award, and the Safford Award, University of Pittsburgh, for Excellence in Teaching. 